\title{
A RISK INDEX TO PREDICT ATRIAL FIBRILLATION AFTER CARDIAC SURGERY
}

\author{
Le Thanh Hung ${ }^{1}$, Abdulmueti Alshareef ${ }^{2}$, Tareq AL-Ahdal ${ }^{3}$, Pham Tho Tuan Anh ${ }^{4}$, Do \\ Quang Huan ${ }^{1}$, Do Van Trang ${ }^{5}$, Sy Hoang ${ }^{6}$, and Nguyen Tien Huy ${ }^{7}$ \\ ${ }^{1}$ Heart Institute, Ho Chi Minh City, Vietnam \\ ${ }^{2}$ University of Zawia College of Human Medicine \\ ${ }^{3}$ Jordan University of Science and Technology \\ ${ }^{4}$ Cho Ray Hospital \\ ${ }^{5}$ Ben Cat Hospital, Binh Duong Province, Vietnam \\ ${ }^{6}$ Ho Chi Minh City Medical and Pharmaceutical University Hospital \\ ${ }^{7}$ Affiliation not available
}

June 3, 2020

\begin{abstract}
Background: Atrial fibrillation is the most common complication after cardiac surgery and is associated with an increased risk of postoperative adverse events. The objective of this study was to develop a risk index to predict atrial fibrillation after cardiac surgery. Methods: We performed a prospective cohort study. A total of 405 patients who had undergone adult cardiac surgery from 2015 September to 2016 August at Heart Institute of HCMC and Cho Ray Hospital. Results: In the overall, 98 patients developed POAF (24.2\%). The risk score included three significant risk factors (age, left atrial diameter $>41 \mathrm{~mm}$, Coronary Artery Bypass Graft with concomitant mitral valve replacement or repair). The point values for were 1 for the age ? 60, 1 for CABG with concomitant mitral valve replacement or repair and 1 for left atrial diameter $>41 \mathrm{~mm}$, and the total risk score ranges from 0 to 3 (AUC $=0.69,95 \%$ CI: $0.63-0.75$ ), the best cutoff point was 1 The incidences of POAF associated with scores were: patient with a score of 0 , predicted probabilities of POAF was $8.6 \%$; a score of $1: 30.1 \%$; a score of 2 : $40.8 \%$; a score of 3: $58.3 \%$. Bootstrapping with 5,000 samples confirmed the final model provided consistent predictions. Conclusions: We developed a simple risk score based on clinical variables and these variables can be collected easily before surgery. This risk score may help accurately stratifies the risk of POAF to identify patients at high risk of POAF before cardiac surgery.
\end{abstract}

\section{Hosted file}

The main document.docx available at https://authorea.com/users/329342/articles/456531-a-riskindex-to-predict-atrial-fibrillation-after-cardiac-surgery 


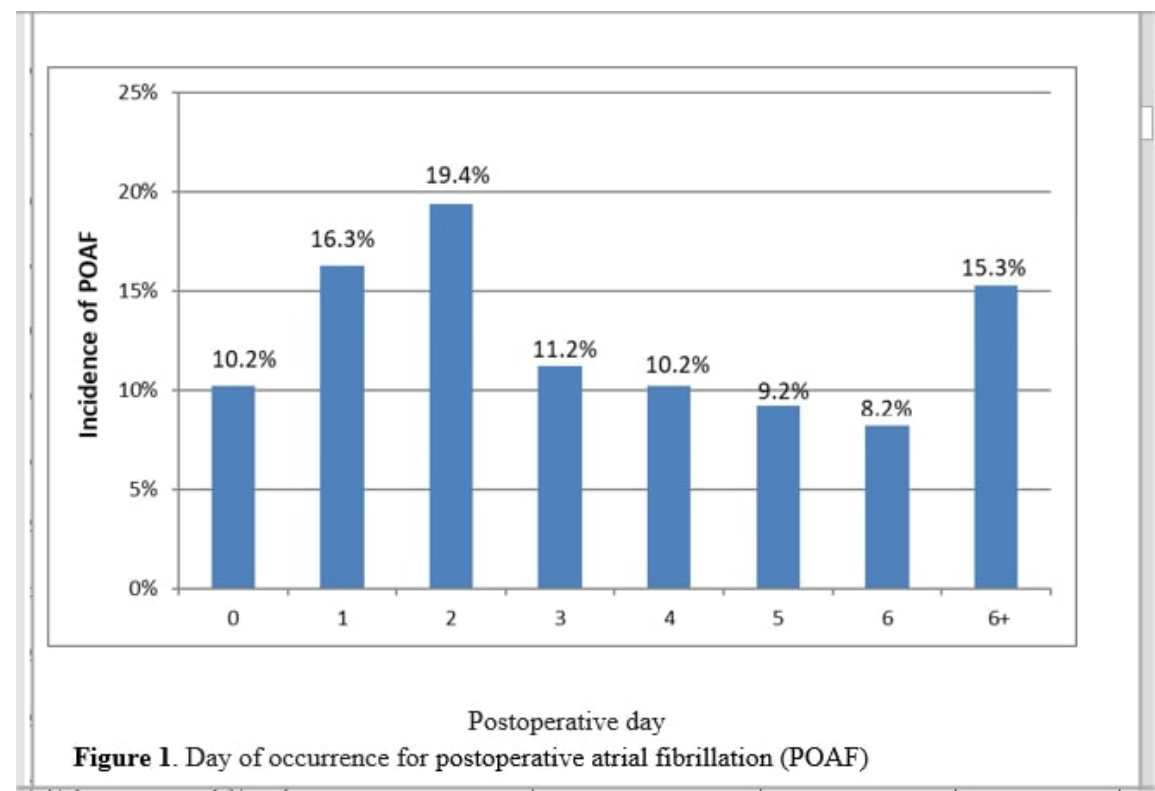

Figure 1. Day of occurrence for postoperative atrial fibrillation (POAF)

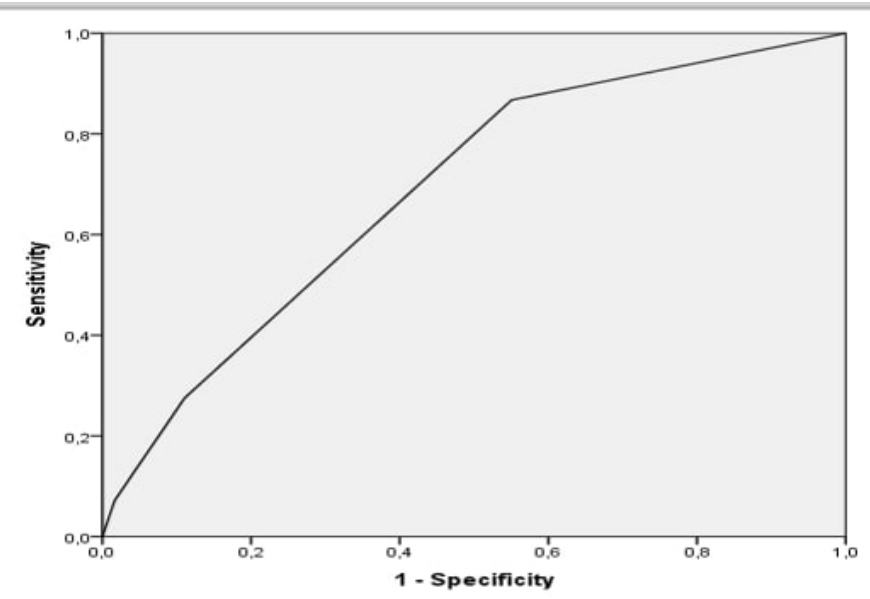

Figure 2. The area under the ROC curve for the logistic regression model predicting POAF is 0.69 , indicating fair discrimination between $\mathrm{POAF}$ and no-POAF 